\title{
EIGENPROBLEMS FROM FINITE ELEMENT ANALYSIS OF FLUID-STRUCTURE INTERACTIONS
}

\author{
LORRAINE OLSON and THOMAS VANDINI \\ Department of Mechanical Engineering and Applied Mechanics, University of Michigan, Ann Arbor, \\ MI 48109-2125, U.S.A.
}

(Received 19 September 1988)

\begin{abstract}
We present two methods for calculating resonant frequencies and mode shapes for fluid-structure vibration problems. We model the fluid-structure interaction problem using the $\phi-U-P_{0}$ finite element technique. This eigenproblem is more complicated than for ordinary structural analyses, as the formulation produces a quadratic eigenproblem (similar to those for gyroscopic systems). We derive properties for this quadratic eigenproblem from an equivalent linear problem. From these properties we develop eigenvalue solution techniques based on the determinant search and subspace iteration procedures.
\end{abstract}

\section{INTRODUCTION}

Fluid-structure interaction problems arise in many engineering systems. Predicting the coupled motions of the fluid and the structure is generally a difficult task. Finite element analysis can be used to predict the response of some simple fluid-structure systems, especially if the fluid is initially at rest and undergoes only small motions. These coupled vibration problems can be solved efficiently using the recently developed $\phi-U-P_{0}$ method [1-3], which is generally quite robust. However, finding the modes and natural frequencies of the system is a somewhat complicated task because of the nature of the finite element matrix equations. The goal of this paper is to describe two robust methods for finding the first few eigenvalues and eigenvectors of large matrix systems derived from the $\phi-U-P_{0}$ method. One of the methods is based on the determinant search technique and the other is based on the subspace iteration technique.

In a previous paper, Olson and Bathe [1] developed a modified determinant search method for the $\phi-U-P_{0}$ technique. The current determinant search approach has two main advantages: the inverse iteration is now derived directly from an equivalent linear eigenproblem so we can guarantee convergence, and the orthogonalization procedure is now exact. (The previous approach used an approximate inverse iteration and orthogonalization.) However, to gain these advantages we must modify the standard determinant search algorithm significantly and include an extra vector in the iteration.

Olson and Bathe did not study the modifications to the subspace iteration technique. This method can be considerably more efficient for large matrix systems, and we investigate modifications to this algorithm as well.

In addition, we derive all of the properties of the eigenproblem from the gyroscopic form of the governing equations. We feel that this gives insight into the underlying physics of the fluid-structure problem.
By taking this approach, we identify a fundamental difficulty with zero eigenvalues which had not been noted previously.

Because of the unique nature of this eigenproblem, there is little other work which directly addresses its efficient solution. However, a number of studies on the quadratic eigenproblem resulting from analysis of spinning structures (a gyroscopic system) take similar approaches. Meirovitch [4, 5], established some of the basic principles for gyroscopic eigenproblems (which in general have complex eigenvectors and purely imaginary eigenvalues), and used these to solve small problems. Gupta [6] presented a combined spectrum slicing and inverse iteration technique which is quite similar to the determinant search technique. Wittrick and Williams [7] used the spectrum slicing technique to find eigenvalues for spinning systems. Huseyin [8], Plaut [9], and Janssens [10] discussed various ways of transforming the gyroscopic eigenproblem into a linear eigenproblem. Borri and Mantegazza [11] and Bauchau [12] discussed modifications of the Lanczos technique for spinning structures. Dietrich [13] discussed gyroscopic problems in the more general context of the skew-symmetric eigenproblem. Fricker [14] studied quadratic eigenproblems in a different context - as a subset of general higher-order eigenproblems arising in dynamic analysis. He developed a variant of subspace iteration which is efficient for small, full matrices.

Section 2 describes the matrix equations and the corresponding eigenproblem for the fluid-structure interaction problem. Useful mathematical properties of the eigenproblem are derived from the gyroscopic form of the governing equations in Sec. 3. In Sec. 4 we outline the specific determinant search procedure used to find the eigenvalues and eigenvectors, and Sec. 5 discusses the modified subspace iteration technique. Section 6 discusses the computational efficiency of the two methods, and Sec. 7 demonstrates the algorithm on the analysis of a plate in water. Section 8 summarizes our results. 


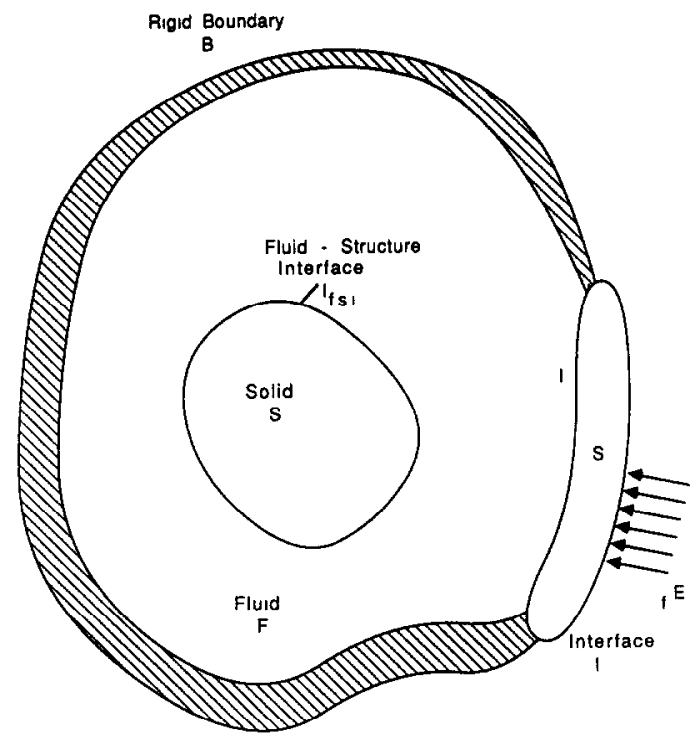

Fig. 1. General fluid-structure domain.

\section{DERIVATION OF THE EIGENPROBLEM}

\subsection{Domain discretization}

Figure 1 shows a general fluid-structure domain. We assume that the solid is linear and elastic and employs displacements $u$ as the nodal variable as usual. In the fluid we make the ordinary acoustic assumptions: the fluid is initially at rest, it is compressible, and it undergoes small isentropic motions. Olson and Bathe [1] described the $\phi-U-P_{0}$ formulation in detail, and we review it briefly here. We express the fluid pressure as $P_{0}-\rho_{F} \phi$, where $P_{0}$ is the static pressure in each fluid region and $\rho_{F} \phi$ is the product of the fluid density and the velocity potential. With this notation, the principle of virtual work for the solid is

$$
\begin{aligned}
\int \overline{\boldsymbol{\epsilon}}^{T} \mathbf{C}_{S} \boldsymbol{\epsilon} \mathrm{d} S & +\int \rho_{S^{\mathbf{u}}}{ }^{\tilde{\mathbf{u}}} \mathrm{d} S \\
& =\int \overline{\mathbf{u}}^{T} \mathbf{f}^{E} \mathrm{~d} I-\int \bar{u}_{n}\left(P_{0}-\rho_{F} \phi\right) \mathrm{d} I_{t s i},
\end{aligned}
$$

where $\bar{x}$ is the virtual $x, \epsilon$ are the strains in the solid, $\mathbf{C}_{S}$ is the stress-strain matrix, $\rho_{S}$ is the density of the solid, $\mathbf{f}^{E}$ are external forces applied to the solid, and $u_{n}$ is the normal displacement of fluid-structure interface (positive into fluid).

For the fluid, we find that

$$
\begin{aligned}
\int \frac{1}{\beta}\left(\overline{P_{0}-\rho_{F} \bar{\phi}}\right)\left(P_{0}-\rho_{F} \phi\right) & \mathrm{d} F-\int \rho_{F}(\nabla \bar{\phi})^{T}(\nabla \phi) \mathrm{d} F \\
& =\int\left(\overline{P_{0} \cdots \rho_{F} \phi}\right) u_{n} \mathrm{~d} I_{t s i},
\end{aligned}
$$

where $\beta$ is the bulk modulus of the fluid.

Using ordinary isoparametric finite elements for u and $\phi$, we find the following matrix equations (see [I] for details):

$$
\begin{aligned}
& {\left[\begin{array}{ccc}
\mathbf{K}_{S S} & \mathbf{0} & \mathbf{K}_{P S}^{T} \\
\mathbf{0} & -\mathbf{K}_{F F} & \mathbf{0} \\
\mathbf{K}_{P S} & \mathbf{0} & \mathbf{K}_{P P}
\end{array}\right]\left[\begin{array}{c}
\mathbf{U} \\
\mathbf{\Phi} \\
\mathbf{P}_{0}
\end{array}\right]} \\
& +\left[\begin{array}{ccc}
\mathbf{0} & \mathbf{C}_{F S}^{T} & \mathbf{0} \\
\mathbf{C}_{\mathrm{FS}} & \mathbf{0} & \mathbf{C}_{P F}^{T} \\
\mathbf{0} & \mathbf{C}_{P F} & \mathbf{0}
\end{array}\right]\left[\begin{array}{c}
\dot{\mathbf{U}} \\
\mathbf{\Phi} \\
\mathbf{P}_{\mathrm{n}}
\end{array}\right] \\
& +\left[\begin{array}{ccc}
\mathbf{M}_{\mathrm{SS}} & \mathbf{0} & \mathbf{0} \\
\mathbf{0} & -\mathbf{M}_{F F} & \mathbf{0} \\
\mathbf{0} & \mathbf{0} & \mathbf{0}
\end{array}\right]\left[\begin{array}{c}
\ddot{\mathbf{U}} \\
\dot{\boldsymbol{\Phi}} \\
\dot{\mathbf{P}}_{\mathrm{n}}
\end{array}\right]=\left[\begin{array}{c}
\mathbf{R}_{S} \\
\mathbf{0} \\
\mathbf{0}
\end{array}\right] .
\end{aligned}
$$

Notice that this matrix equation is applicable to general transient analysis, and that the $\mathbf{C}$ matrices represent coupling between various degrees of freedom rather than damping (since the fluid is assumed to be inviscid). $\mathbf{K}_{s s}$ is the ordinary structural stiffness matrix, and $\mathbf{K}_{F F}$ is the kinetic energy matrix for the fluid. $\mathbf{K}_{P P}$ represents the bulk stiffness of the fluid in static analysis. $\mathbf{K}_{P S}$ couples the static pressure to all displacements on the fluid-structure interface. $\mathbf{C}_{F T}$ couples the static pressure to all velocity potential degrees of freedom. $\mathbf{C}_{F S}$ couples the velocity potential to displacements on the fluid-structure boundary. $\mathbf{M}_{s S}$ is the ordinary structural stiffness matrix and $\mathbf{M}_{F F}$ is the potential energy matrix for the fluid.

\subsection{Condensation of $\mathbf{P}_{0}$}

We can actually eliminate the $P_{0}$ degrees of freedom completely from the transient matrix equations as suggested by Parlett (personal communication). We find

$$
\mathbf{P}_{0}=-\mathbf{K}_{P P}^{-1}\left(\mathbf{K}_{P S} \mathbf{U}+\mathbf{C}_{P F} \boldsymbol{\phi}\right)
$$

so that

$$
\begin{aligned}
{\left[\begin{array}{cc}
\mathbf{R}_{S S} & \mathbf{0} \\
\mathbf{0} & \mathbf{K}_{F F}
\end{array}\right]\left[\begin{array}{l}
\mathbf{U} \\
\mathbf{\Phi}
\end{array}\right]+\left[\begin{array}{cc}
\mathbf{0} & \mathbf{C}_{F S}^{T} \\
-\hat{\mathbf{C}}_{F S} & \mathbf{0}
\end{array}\right]\left[\begin{array}{l}
\mathbf{U} \\
\boldsymbol{\phi}
\end{array}\right] } \\
+\left[\begin{array}{cc}
\mathbf{M}_{S S} & \mathbf{0} \\
\mathbf{0} & \hat{\mathbf{M}}_{F F}
\end{array}\right]\left[\begin{array}{l}
\mathbf{U} \\
\ddot{\boldsymbol{\Phi}}
\end{array}\right]=\left[\begin{array}{c}
\mathbf{R}_{S} \\
\mathbf{0}
\end{array}\right],
\end{aligned}
$$

where

$$
\begin{gathered}
\mathbf{R}_{S S}=\mathbf{K}_{S S}-\mathbf{K}_{P S}^{T} \mathbf{K}_{P P}^{-1} \mathbf{K}_{P S} \\
\hat{\mathbf{C}}_{F S}=\mathbf{C}_{F S}-\mathbf{C}_{P F}^{T} \mathbf{K}_{P P}^{-1} \mathbf{K}_{P S} \\
\hat{\mathbf{M}}_{F F}=\mathbf{M}_{F F}+\mathbf{C}_{P F}^{T} \mathbf{K}_{P P}^{-1} \mathbf{C}_{P F}
\end{gathered}
$$

Now the system is a 'conservative gyroscopic system" as discussed by Meirovitch [15]. The stiffness and mass matrices are much simpler, and it can be shown that $\mathbf{K}_{F F}, \mathbf{M}_{S S}$ and $\hat{\mathbf{M}}_{F F}$ are positive definite. (We assume that $\widehat{\mathbf{R}}_{s S}$ is also positive definite; see Sec. 3.) However, now $\hat{\mathbf{R}}_{s S}$ and $\hat{\mathbf{M}}_{F F}$ are essentially full. 
Therefore we see that condensing out $P_{0}$ produces a useful conceptual result, but it is not as useful from a computational point of view.

\subsection{Corresponding eigenproblem}

Let us express the motions of the system as

$$
\left[\begin{array}{l}
\mathbf{U} \\
\mathbf{\Phi}
\end{array}\right]=\left[\begin{array}{l}
\mathbf{U} \\
\tilde{\mathbf{\Phi}}
\end{array}\right] \mathrm{e}^{-\lambda \ell} \text {. }
$$

With this assumption, both the mode shape and the frequency may be complex. Inserting this into the transient equations of motion, and setting the forcing vector to zero, yields

$$
\begin{array}{r}
{\left[\begin{array}{cc}
\mathbf{R}_{s S} & \mathbf{0} \\
\mathbf{0} & \mathbf{K}_{F F}
\end{array}\right]\left[\begin{array}{l}
\mathbf{U} \\
\boldsymbol{\Phi}
\end{array}\right]-\lambda\left[\begin{array}{cc}
\mathbf{0} & \hat{\mathbf{C}}_{F S}^{T} \\
-\hat{\mathbf{C}}_{F S} & \mathbf{0}
\end{array}\right]\left[\begin{array}{l}
\mathbf{U} \\
\boldsymbol{\Phi}
\end{array}\right]} \\
+\lambda^{2}\left[\begin{array}{cc}
\mathbf{M}_{s S} & \mathbf{0} \\
\mathbf{0} & \overline{\mathbf{M}}_{F F}
\end{array}\right]\left[\begin{array}{l}
\mathbf{U} \\
\boldsymbol{\Phi}
\end{array}\right]=\left[\begin{array}{l}
0 \\
\mathbf{0}
\end{array}\right]
\end{array}
$$

However, as this is a gyroscopic system [15], the eigenvalues $\lambda$ of this matrix system must be pure imaginary. In addition, the solution for $\mathbf{U}$ and $\boldsymbol{\phi}$ will be $90^{\circ}$ out of phase. $\dagger$ Therefore, we can change our assumption for the modal displacements to

$$
\left[\begin{array}{l}
\mathbf{U} \\
\mathbf{\Phi}
\end{array}\right]=\left[\begin{array}{l}
\mathbf{U} \sin \omega t \\
\hat{\mathbf{\Phi}} \cos \omega t
\end{array}\right]
$$

which yields a matrix equation of the form

$$
\begin{array}{r}
{\left[\begin{array}{cc}
\mathbf{R}_{S S} & \mathbf{0} \\
\mathbf{0} & \mathbf{K}_{F F}
\end{array}\right]\left[\begin{array}{l}
\mathbf{U} \\
\boldsymbol{\Phi}
\end{array}\right]-\omega\left[\begin{array}{cc}
\mathbf{0} & \mathbf{C}_{F S}^{T} \\
\mathbf{C}_{F S} & \mathbf{0}
\end{array}\right]\left[\begin{array}{l}
\mathbf{0} \\
\boldsymbol{\Phi}
\end{array}\right]} \\
-\omega^{2}\left[\begin{array}{cc}
\mathbf{M}_{S S} & \mathbf{0} \\
\mathbf{0} & \mathbf{M}_{F F}
\end{array}\right]\left[\begin{array}{l}
\mathbf{0} \\
\boldsymbol{\Phi}
\end{array}\right]=\left[\begin{array}{l}
\mathbf{0} \\
\mathbf{0}
\end{array}\right],
\end{array}
$$

or,

$$
\mathbf{K X}-\omega \mathbf{C X}-\omega^{2} \mathbf{M X}=0
$$

Therefore we have a real symmetric eigenproblem which is quadratic in the real eigenvalues $\omega$ and which has real eigenvectors $\mathbf{X}$.

\section{PROPERTIES OF THE EIGENPROBLEM}

To understand the properties of this eigenvalue problem, let us define $\mathbf{Z}=\omega \mathbf{X}$ and consider the equivalent eigenproblem:

$$
\left[\begin{array}{cc}
\mathbf{K} & \mathbf{0} \\
\mathbf{0} & \mathbf{M}
\end{array}\right]\left[\begin{array}{l}
\mathbf{X} \\
\mathbf{Z}
\end{array}\right]=\omega\left[\begin{array}{cc}
\mathbf{C} & \mathbf{M} \\
\mathbf{M} & \mathbf{0}
\end{array}\right]\left[\begin{array}{l}
\mathbf{X} \\
\mathbf{Z}
\end{array}\right]
$$

We assume that there are no rigid body modes in the system, so that the matrix formed from $K$ and $M$ is positive definite.

$\dagger$ One will be purely imaginary and the other will be purely real. This phase difference physically occurs because we require $\dot{u}_{n} \approx \partial \phi / \partial n$ on the fluid-structure interface.
(1) Real eigenvalues. As discussed in Sec. 2, the eigenvalues $\omega$ of the fluid-structure eigenproblem are purely real. The eigenvalues must be real, as there is no dissipation (or sinks or sources) in the physical problem.

(2) Eigenvalue/eigenvector pairs. Since eqn (13) is quadratic in $\omega$, we see that the system must have $2 N$ roots $\omega$. If we suppose that $\omega$ is the eigenvalue associated with the eigenvector $\mathbf{X}^{T}=\left[\begin{array}{ll}\mathbf{U} & \boldsymbol{\Phi}\end{array}\right]$, then we see by direct substitution that $-\omega$ and $\mathbf{X}^{T}=\left[\begin{array}{ll}\mathbf{U} & -\Phi\end{array}\right]$ is also a solution. Therefore we only solve for the $N$ positive eigenvalues.

(3) Characteristic polynomial. As would be expected, the characteristic polynomial of the system is

$$
\operatorname{det}\left(\mathbf{K}-\mu \mathbf{C}-\mu^{2} \mathbf{M}\right) \equiv \operatorname{det}\left(\mathbf{K}^{*}\right)=0
$$

(4) Spectrum slicing. We find the following result for our quadratic eigenvalue problem:

If we decompose $\mathbf{K}^{*}$ into $\mathbf{L D L}^{T}$ ( $\mathrm{L}$ is a lower triangular matrix and $\mathbf{D}$ is a diagonal matrix), then the number of negative elements in $\mathbf{D}$ is equal to the number of eigenvalues less than $\mu$.

This result is derived in the Appendix. Wittrick and Williams [7] derived this property in some detail for the general gyroscopic eigenproblem.

(5) Shifting. We may shift the eigenvalues of the linear problem in the ordinary way:

$$
\left[\begin{array}{cc}
\mathbf{K}-\mu \mathbf{C} & -\mu \mathbf{M} \\
-\mu \mathbf{M} & \mathbf{M}
\end{array}\right]\left[\begin{array}{l}
\mathbf{X} \\
\mathbf{Z}
\end{array}\right]=(\omega-\mu)\left[\begin{array}{cc}
\mathbf{C} & \mathbf{M} \\
\mathbf{M} & 0
\end{array}\right]\left[\begin{array}{l}
\mathbf{X} \\
\mathbf{Z}
\end{array}\right] .
$$

For iteration purposes, we can rewrite this equation as

$$
\left[\begin{array}{ll}
\mathbf{K}^{*} & \mathbf{0} \\
\mu \mathbf{I} & \mathbf{I}
\end{array}\right]\left[\begin{array}{l}
\mathbf{X} \\
\mathbf{Z}
\end{array}\right]=(\omega-\mu)\left[\begin{array}{cc}
\mathbf{C}+\mu \mathbf{M} & \mathbf{M} \\
\mathbf{I} & \mathbf{0}
\end{array}\right]\left[\begin{array}{l}
\mathbf{X} \\
\mathbf{Z}
\end{array}\right] .
$$

(6) Rayleigh quotient. We may compute a Rayleigh quotient, or eigenvalue estimate, from the linear problem:

$$
\frac{1}{\omega} \approx \frac{\mathbf{X}^{T} \mathbf{C X}+\mathbf{X}^{T} \mathbf{M Z}+\mathbf{Z}^{T} \mathbf{M X}}{\mathbf{X}^{T} \mathbf{K X}+\mathbf{Z}^{T} \mathbf{M Z}}
$$

Note that the denominator is always positive. Naturally, other estimates are possible.

(7) Orthogonality relationships. Two orthogonality relationships may be derived from the linear problem. We find that

$$
\mathbf{X}_{i}^{T} \mathbf{K} \mathbf{X}_{j}+\mathbf{Z}_{i}^{T} \mathbf{M} \mathbf{Z}_{j}=\omega_{i} \delta_{i j}
$$

and

$$
\mathbf{X}_{i}^{T} \mathbf{C X}+\mathbf{X}_{i}^{T} \mathbf{M} \mathbf{Z}_{j}+\mathbf{Z}_{i}^{T} \mathbf{M} \mathbf{X}_{j}=\delta_{y} .
$$

$\delta_{y}$ is the Kronecker delta. 
(8) Gram-Schmidt orthogonalization. In the determinant search algorithm, we use Gram-Schmidt orthogonalization [16] to make eigenvector estimates $\mathbf{X}$ and $\mathbf{Z}$ orthogonal to all previously calculated eigenvectors $\mathbf{X}_{i}$. Using the second orthogonality relationship above (20), we find that the revised eigenvector estimates $\mathbf{X}$ and $\mathbf{Z}$ are

$$
\begin{gathered}
\mathbf{X}=\mathbf{X}-\sum \alpha_{1} \mathbf{X}_{t} \\
\mathbf{Z}=\mathbf{Z}-\sum \alpha_{i} \omega_{i} \mathbf{X}_{t},
\end{gathered}
$$

where

$$
\alpha_{2}=\mathbf{X}^{T} \mathbf{C X}+\mathbf{X}^{T} \mathbf{M}\left(\omega_{t} \mathbf{X}_{i}\right)+\mathbf{Z}^{T} \mathbf{M} \mathbf{X}_{t} .
$$

Notice that, as we will be iterating on $\mathbf{Z}$ as well as $X$, we can orthogonalize exactly. In the previous paper [1], we used $\bar{\omega} \mathbf{X}$ in place of $\mathbf{Z}$ in eqn (23). Unfortunately, this is often a poor estimate of $\mathbf{Z}$ except when the inverse iteration nears convergence. In addition, with $\bar{\omega} \overline{\mathbf{X}}$ instead of $\mathbf{Z}$, eqn (21) conflicts with eqn (22).

\subsection{Difficulties because of rigid body modes}

If $\mathbf{R}_{s s}$ is not positive definite, there may be rigid body modes in the solid. In that case, we cannot separate the rigid body modes from the other eigen. vectors. For example, suppose that $\mathbf{X}_{1}$ is a rigid body mode $\left(\mathbf{Z}_{1}=0\right)$ and we have an approximation to the second eigenvector. $\overline{\mathbf{X}}_{2}$ and $\mathbf{Z}_{2}$. Set

$$
\begin{gathered}
\mathbf{X}_{2}=\overline{\mathbf{X}}_{2}-\alpha_{1} \mathbf{X}_{1} \\
\mathbf{Z}_{2}=\overline{\mathbf{Z}}_{2} .
\end{gathered}
$$

To make $\mathbf{X}_{2}$ and $\mathbf{Z}_{2}$ orthogonal to the rigid body mode, we require that

$$
\mathbf{X}_{2}^{T} \mathbf{C X}_{1}+\mathbf{X}_{2}^{T} \mathbf{M Z}_{1}+\mathbf{Z}_{2}^{T} \mathbf{M} \mathbf{X}_{1}=0
$$

to get

$$
\mathbf{X}_{2}^{T} \mathbf{C X}-\alpha_{1} \mathbf{X}_{1}^{T} \mathbf{C X}+\mathbf{Z}_{2}^{T} \mathbf{M} \mathbf{X}_{1}=0
$$

However, the rigid body mode only involves the solid so that $\mathbf{X}_{1}^{T} \mathbf{C X _ { 1 }}=0$. As a result, we cannot calculate $\alpha_{1}$ to perform the orthogonalization. Similar problems arise if we attempt to use the first orthogonality relationship (19).

Note that this does not mean that an inverse iteration with a shift will not converge to the correct root, it simply may not.

\subsection{Inclusion of $\mathbf{P}_{0}$}

Each of the properties discussed above was derived for the case of positive definite $\mathbf{K}$ and $\mathbf{M}$, which results when $P_{0}$ is eliminated. It can be shown that only properties (2) and (4) require modification when the pressure variables are not condensed out. Let $N_{p}$ represent the number of pressure degrees of freedom. Property (2) becomes

(2') There are $2\left(N-N_{p}\right)$ finite eigenvalue/ eigenvector pairs which solve the problem.

Property (4), the spectrum slicing rule, becomes

(4) If we decompose $\mathbf{K}^{*}$ into $\mathbf{L D L}^{T}$, then the number of negative elements in D minus $N_{n}$ is equal to the number of eigenvalues less than $\mu$.

This modification arises from the fact that $\mathbf{K}_{p p}$ is a diagonal matrix containing all negative numbers.

\section{DETERMINANT SEARCH TECHNIQUE}

Having discussed some of the properties of the quadratic fluid-structure interaction eigenproblem, we can now describe a procedure for finding the eigenvalues of large systems. This procedure is based on the determinant search technique [16] for the equivalent linear eigenproblem.

First, we must count the number of pressure degrees of freedom (by counting the number of negative elements in the unfactored stiffness matrix) and verify that there are no zero eigenvalues in the system by factorizing the stiffness matrix. Next, for each eigenvalue in turn, we

(1) Iterate on characteristic polynomial until near an eigenvalue. This is a simple secant iteration, just as in ordinary determinant search routines (see [16]). We stop when our guess for a root $\mu$ is near an actual root or when we have jumped over a root.

(2) Perform inverse iteratıon with a shift. We use eqn (17) as a starting point, to give

$$
\left[\begin{array}{ll}
\mathbf{K}^{*} & \mathbf{0} \\
\mu \mathbf{I} & \mathbf{I}
\end{array}\right]\left[\begin{array}{l}
\mathbf{X}^{K+1} \\
\mathbf{Z}^{K+1}
\end{array}\right]=\left[\begin{array}{cc}
\mathbf{C}+\mu \mathbf{M} & \mathbf{M} \\
\mathbf{I} & \mathbf{0}
\end{array}\right]\left[\begin{array}{l}
\mathbf{X}^{K} \\
\mathbf{Z}^{K}
\end{array}\right] .
$$

In each iteration, the current eigenvector estumate is orthogonalized against the last seven previously calculated eigenvectors. Table 1 shows the specific algorithm used.

Finally, we use spectrum shicing to verify that no roots have been skipped.

\section{SUBSPACE ITERATION TECHNIQUE}

For eigenproblems with many degrees of freedom, subspace iteration techniques [16] are generally considercd to be faster. Subspace iteration is related to inverse iteration, so our subspace iteration procedure is similar to the algorithm outlined in Table 1.

As in the determinant search algorithm, we first count the number of pressure degrees of freedom and verify that there are no zero eigenvalues in the system.

Next, we choose a set of $q$ trial vectors to initialize the eigenvectors for the iteration. We use trial vectors 
Table 1. Steps in inverse iteration for determinant search routine

Initialization

$$
\begin{gathered}
\text { Set } \mathbf{X} \text { so that } X_{1}=i \\
\text { Set } \mathbf{Z}=\mathbf{X} \\
\mathbf{Y}=\mathbf{M X} \\
B O T=\mathbf{X}^{T} \mathbf{Y} \\
\mathbf{Y}=2 \mu \mathbf{Y}+\mathbf{C X}
\end{gathered}
$$

Iteration

$$
\begin{gathered}
\text { Solve } \mathbf{K}^{*} \mathbf{X}=\mathbf{Y} \\
\mathbf{Z}=\mathbf{Z}+\mu \mathbf{X} \\
B O T=B O T+\mathbf{X}^{T} \mathbf{Y} \\
\mathbf{Y}=\mathbf{M Z} \\
T O P=\mathbf{Y}^{T} \mathbf{X} \\
\mathbf{Y}=\mathbf{Y}+\mathbf{C X} \\
T O P=T O P+\mathbf{X}^{T} \mathbf{Y} \\
R Q=T O P /(B O T+\mu T O P)
\end{gathered}
$$

If converged, skip to converged solution

$$
\begin{gathered}
\alpha_{j}=\mathbf{X}^{T}(\mathbf{C X})+\omega_{j} \mathbf{X}^{T}\left(\mathbf{M X} \mathbf{X}_{\jmath}\right)+\mathbf{Z}^{T}(\mathbf{M X},) \\
\mathbf{Y}=\left[\mathbf{Y}-\sum \boldsymbol{x}_{,}(\mathbf{C X},)-\sum \alpha_{l} \omega_{l}(\mathbf{M X},)\right] / \sqrt{ }(|T O P|) \\
\mathbf{X}=\left[\mathbf{X}-\sum \alpha_{j} \mathbf{X}_{j}\right] / \sqrt{ }(|T O P|) \\
\mathbf{Z}=\mathbf{M X} \\
\mathbf{Y}=\mathbf{Y}+\mu \mathbf{Z} \\
B O T=\mathbf{X}^{T} \mathbf{Z} \\
\mathbf{Z}=\mathbf{X}
\end{gathered}
$$

Iterate again
First part of forcing function

First part of bottom of Rayleigh quotient

Rest of forcing function

Form new $\mathbf{X}$

Finish forming new $\mathbf{Z}$

Finish forming bottom of Rayleigh quotient First part of forcing function for next iteration First part of top of Rayleigh quotient

Second part of forcing function for next iteration

Finish forming top of Rayleigh quotient

Test Rayleigh quotient for convergence

Perform Gram-Schmidt orthogonalization

on last seven eigenvectors

and scale $\mathbf{Y}$ and $\mathbf{X}$

Finish forming forcing function

( $Z$ is a dummy vector here)

First part of Rayleigh quotient for next iteration First part of new $\mathbf{Z}$

Converged solution

$$
\begin{gathered}
\mathbf{X}=\mathbf{X} / \sqrt{ }(\mid \text { TOP } \mid) \\
\text { Store } \mathbf{X}, \mathbf{C X}, \mathbf{M X}, \omega
\end{gathered}
$$

which are similar to those used by Bathe [16] for the problem without the coupling matrix $\mathbf{C}$ :

(1) $\mathbf{X}_{1}$ is a vector containing the inverse of the diagonal of $K \cdot Z_{1}$ contains the inverse of the diagonal of $\mathbf{M}$. If $K_{u}$ is zero, we set that entry of $\mathbf{X}_{\mathbf{1}}$ to zero. If $M_{i i}$ is zero, we assume that we have found a pressure degree of freedom. Therefore we change the sign on the corresponding $X_{1}$ entry and set the $Z_{1}$ entry equal to the average $K_{i l} / M_{i j}$ times $\mathbf{X}_{1} \cdot \mathbf{X}_{2}=\mathbf{X}_{1}$ and $\mathbf{Z}_{2}=-\mathbf{Z}_{1}$.

(2) $\mathbf{X}_{3}$ is zero, except that it has a $1 / \sqrt{ }\left(K_{i i}\right)$ at the position corresponding to the minimum $\sqrt{ }\left(K_{i i} / M_{i l}\right)$. $\mathbf{Z}_{3}=\sqrt{ }\left(\boldsymbol{K}_{i t} / \boldsymbol{M}_{i t}\right) \mathbf{X}_{3} . \mathbf{X}_{4}=\mathbf{X}_{3}$ and $\mathbf{Z}_{4}=-\mathbf{Z}_{3}$.

(3) $\mathbf{X}_{5}, \mathbf{Z}_{5}, \mathbf{X}_{6}, \mathbf{Z}_{6}$ are formed like 3 and 4 , but using the second-smallest value of $K_{i i} / M_{t i}$. Subsequent trial vectors follow this pattern.

As we know that the eigenvectors for $+\omega$ and $-\omega$ are related, we also choose our eigenvectors in pairs. These choices appeared to work reasonably well.

Now we may begin the subspace iteration. Note that now $\mathbf{X}$ with no subscript represents the entire set of trial vectors $\left(\mathbf{X}=\left[\begin{array}{llll}\mathbf{X}_{1} & \mathbf{X}_{2} & \mathbf{X}_{3} \ldots\end{array}\right]\right)$. The basic iteration procedure has four steps:
Scale eigenvector Save values for future use

(1) Perform one iteration with a shift. Produce a revised estimate for the eigenvector by solving

$$
\left[\begin{array}{ll}
\mathbf{K}^{*} & \mathbf{0} \\
\mu \mathbf{I} & \mathbf{I}
\end{array}\right]\left[\begin{array}{l}
\mathbf{X}^{K+1} \\
\mathbf{Z}^{K+1}
\end{array}\right]=\left[\begin{array}{cc}
\mathbf{C}+\mu \mathbf{M} & \mathbf{M} \\
\mathbf{I} & \mathbf{0}
\end{array}\right]\left[\begin{array}{l}
\mathbf{X}^{K} \\
\mathbf{Z}^{K}
\end{array}\right] .
$$

(2) Project $\mathbf{K}, \mathbf{C}$ and $\mathbf{M}$ onto the subspace. We form small matrices $\mathbf{A}$ and $\mathbf{B}$ as follows:

$$
\begin{gathered}
\mathbf{A}=\left(\mathbf{X}^{K+1}\right)^{T} \mathbf{K} \mathbf{X}^{K+1}+\left(\mathbf{Z}^{K+1}\right)^{T} \mathbf{M} \mathbf{Z}^{K+1} \\
\mathbf{B}=\left(\mathbf{X}^{K+1}\right)^{T} \mathbf{C X}^{K+1}+\left(\mathbf{Z}^{K+1}\right)^{T} \mathbf{M} \mathbf{X}^{K+1} \\
+\left(\mathbf{X}^{K+1}\right)^{T} \mathbf{M} \mathbf{Z}^{K+1}
\end{gathered}
$$

(3) Solve the following projected eigenproblem. Find all the eigenvectors $\mathbf{V}$ and the matrix of eigenvalues $\boldsymbol{\Omega}$ for the small eigenproblem

$$
\mathbf{B V}=\mathbf{A V} \mathbf{\Omega}^{-1}
$$

Note that these eigenvalues are the eigenvalues of the original problem as well, and may be used to check for convergence of the iteration. 
Table 2. Steps in subspace iteration

Initialization

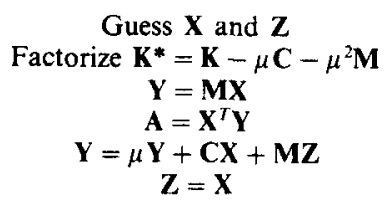

As described in text

First part of forcing function

First part of small eigenproblem

Rest of forcing function

Begin computation of new $\mathbf{Z}$

Iteration

$$
\begin{aligned}
& \text { Solve } K^{*} \mathbf{X}=\mathbf{Y} \\
& \mathbf{Z}=\mathbf{Z}+\mu \mathbf{X} \\
& \begin{array}{c}
\mathbf{A}=\mathbf{A}+\mathbf{X}^{T} \mathbf{Y} \\
\mathbf{Y}=\mathbf{M Z}
\end{array} \\
& \mathbf{B}=\mathbf{Y}^{\boldsymbol{T}} \mathbf{X} \\
& \mathbf{Y}=\mathbf{Y}+\mathbf{C X} \\
& \mathbf{B}=\mathbf{B}+\mathbf{X}^{\prime} \mathbf{Y} \\
& \mathbf{A}=\mathbf{A}+\mu \mathbf{B}
\end{aligned}
$$

Solve $\mathbf{B V}=\mathbf{A V} \boldsymbol{\Omega}^{-1}$

Check eigenvalues for convergence

If converged, skip to converged solution

$$
\begin{gathered}
\mathbf{X}=\mathbf{X V} \\
\mathbf{Y}=\mathbf{Y} \mathbf{V} \\
\mathbf{Z}=\mathbf{M} \mathbf{X} \\
\mathbf{A}=\mathbf{X}^{\top} \mathbf{Z} \\
\mathbf{Y}=\mathbf{Y}+\mu \mathbf{Z} \\
\mathbf{Z}=\mathbf{X}
\end{gathered}
$$

Iterate agaın
Form new $\mathbf{X}$

Finish forming new $\mathbf{Z}$

Second part of $\mathbf{A}$

First part of forcing function for next iteration

First part of $\mathbf{B}$

Second part of forcing function for next iteration

Finish forming $\mathbf{B}$

Finally make A positive definte

Solvc projected eigenproblem

Find an improved $\mathbf{X}$

( $\mathrm{Z}$ is a dummy vector here)

First part of $\mathbf{A}$ for next iteration

Complete forcing function for next iteration

First part of $\mathbf{Z}$ for next iteration
(4) Find an improved approximation to the eigenvectors:

$$
\begin{aligned}
& \mathbf{X}^{K+1}=\mathbf{X}^{K+1} \mathbf{V} \\
& \mathbf{Z}^{K+1}=\mathbf{Z}^{K+1} \mathbf{V}
\end{aligned}
$$

Table 2 shows the specific iteration algorithm used in the computer implementation.

As a final check, we use spectrum slicing to verify that no roots have been skipped.

\section{COMPUTATIONAL EXPENSE}

For a standard determinant search algorithm, the number of operations required for each secant iteration is $O\left(\mathrm{Nm}^{2}\right)$ and for each inverse iteration is $O(N m)$. Here $N$ is the number of degrees of freedom in the system, and $m$ is the mean half bandwidth of the system. For our modified algorithm, the order of growth is the same, although the actual number of operations is higher because of the presence of the $\mathbf{C}$ matrix. For large systems of equations, obviously the $O\left(\mathrm{Nm}^{2}\right)$ term dominates.

For subspace iteration, the order of growth is $O(N m q)$, where $q$ is the number of trial vectors. As the system becomes large, $q$ may be many times smaller than $m$ and a subspace iteration is much faster than determinant search.

\section{IMPLEMENTATION PLATE IN WATER EXAMPLE}

We have verified the implementation of this modified determinant search technique on numerous simple test cases (including the 2-D cases from [1]). As an example of a more difficult analysis, we present the 2-D 'plate in water' test case.

Figure 2 shows the physical system. As the plate in vacuum has a vibration frequency of $2708 \mathrm{rad} / \mathrm{sec}$, we would expect the first resonance for the plate in water to be lower because of the added mass effect. Other modes occur as a result of the resonance of the water in the box (the first acoustic mode for the box with no plate occurs at $7873 \mathrm{rad} / \mathrm{sec}$ ).

We analyzed the system with a coarse mesh, and then successively refined the mesh to obtain larger systems of equations. Figure 3 shows the first two finite element discretizations of the problem. Table 3 shows the calculated values for the first ten frequencies of each mesh. Table 4 gives the number of degrees of freedom and bandwidth for each mesh.

Tables 5 and 6 show the number of iterations required to solve for the first two roots of the system (to a relative accuracy of $10^{-6}$ ) using the determinant 


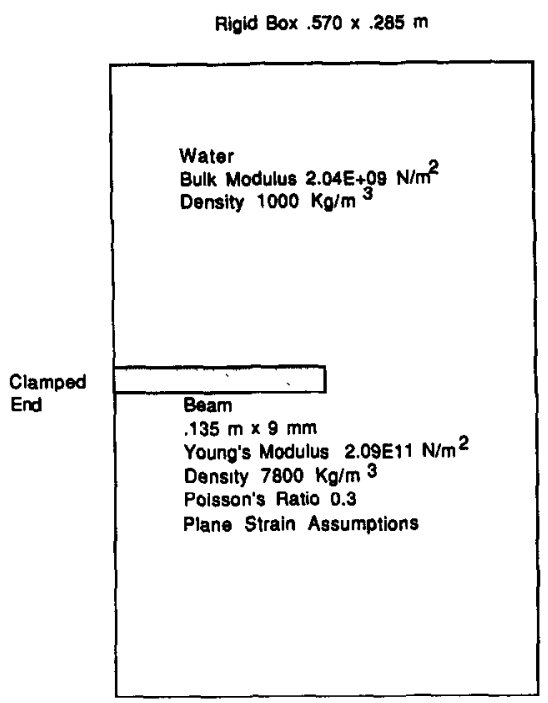

Fig. 2. Plate in water test case.

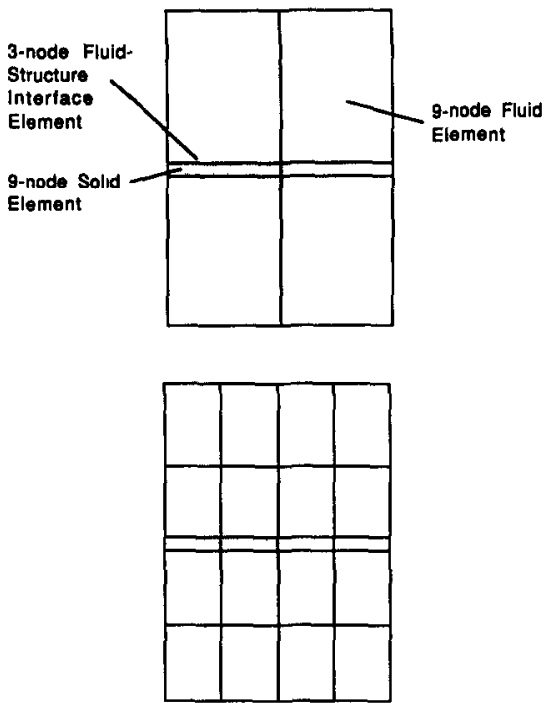

Fig. 3. First two finite element meshes for plate in water test case.

Table 3. First 10 frequencies for plate in water with various meshes

\begin{tabular}{ccccccccccc}
\hline & \multicolumn{10}{c}{ Frequency (rad/sec) } \\
\cline { 2 - 11 } $\begin{array}{c}\text { Elements } \\
\text { in plate }\end{array}$ & $\omega_{1}$ & $\omega_{2}$ & $\omega_{3}$ & $\omega_{4}$ & $\omega_{5}$ & $\omega_{6}$ & $\omega_{7}$ & $\omega_{8}$ & $\omega_{9}$ & $\omega_{10}$ \\
\hline 1 & 2432 & 7210 & 15,804 & 16,976 & 17,419 & 22,773 & 23,547 & 30,248 & 34,733 & 35,509 \\
2 & 1819 & 7137 & 15,719 & 15,811 & 15,950 & 18,498 & 22,404 & 23,792 & 27,736 & 31,590 \\
4 & 1674 & 7055 & 12,112 & 15,706 & 15,904 & 17,511 & 22,362 & 22,442 & 27,124 & 31,479 \\
8 & 1633 & 7033 & 11,594 & 15,705 & 15,900 & 17,477 & 22,342 & 22,358 & 27,004 & 31,471 \\
16 & 1619 & 7030 & 11,511 & 15,704 & 15,900 & 17,471 & 22,325 & 22,357 & 26,985 & 31,470 \\
\hline
\end{tabular}

Table 4. $N$ and $m$ for various meshes

\begin{tabular}{ccc}
$\begin{array}{c}\text { Elements } \\
\text { in plate }\end{array}$ & $\begin{array}{c}\text { Degrees } \\
\text { of freedom } \\
N\end{array}$ & $\begin{array}{c}\text { Mean } \\
\text { bandwidth } \\
m\end{array}$ \\
\hline 1 & 45 & 10 \\
2 & 120 & 17 \\
4 & 363 & 29 \\
8 & 1235 & 56 \\
16 & 4515 & 110 \\
\hline
\end{tabular}

search and subspace iteration techniques. Computation times on an Apollo DN4000 computer are given for reference purposes in these tables. Results for the first 10 roots are shown in Table 7 . When calculating the first two modes of the system, the subspace iteration and determinant search approaches take roughly the same amount of time for the 16-element plate case; for larger systems the subspace iteration would be faster. If we apply a constant shift $\mu=4000 \mathrm{rad} / \mathrm{sec}$ (a value midway between $\omega_{1}$ and $\omega_{2}$ ), we may cut the number of trial vectors for the subspace iteration in half and it becomes faster than determinant search when there are only two elements in the plate. In searching for the first 10 roots, subspace iteration with a $15,000 \mathrm{rad} / \mathrm{sec}$ shift is faster than determinant search for the eight-element beam case. Note that using a constant shift with the subspace iteration can speed up the calculations significantly if the shift value is in the range of the actual eigenvalues for the problem.

\section{SUMMARY AND FUTURE WORK}

We have developed modified determinant search and subspace iteration techniques for finding the first

Table 5. Iterations required to find two roots for plate in water with determinant search

\begin{tabular}{ccccccr}
\hline & \multicolumn{2}{c}{ Mode 1 } & \multicolumn{2}{c}{ Mode 2 } & Total \\
\cline { 2 - 3 } \cline { 5 - 6 } $\begin{array}{c}\text { Elements } \\
\text { in plate }\end{array}$ & $\begin{array}{c}\text { Secant } \\
\text { iterations }\end{array}$ & $\begin{array}{c}\text { Inverse } \\
\text { iterations }\end{array}$ & & $\begin{array}{c}\text { Secant } \\
\text { iterations }\end{array}$ & $\begin{array}{c}\text { Inverse } \\
\text { iterations }\end{array}$ & $\begin{array}{c}\text { time } \\
\text { (sec) }\end{array}$ \\
\hline 1 & 5 & 3 & 7 & 3 & 5 \\
2 & 4 & 3 & 8 & 3 & 25 \\
4 & 4 & 3 & 10 & 3 & 177 \\
8 & 5 & 3 & 10 & 3 & 1762 \\
16 & 5 & 3 & 11 & 3 & 21,938 \\
\hline
\end{tabular}


Table 6. Iterations required to find two roots for plate in water with subspace iteration

\begin{tabular}{|c|c|c|c|c|}
\hline \multirow{2}{*}{$\begin{array}{l}\text { Elements } \\
\text { in plate }\end{array}$} & \multicolumn{2}{|c|}{$\begin{array}{c}\text { No shift } \mu=0 \mathrm{rad} / \mathrm{sec} \\
q=8 \text { trial vectors }\end{array}$} & \multicolumn{2}{|c|}{$\begin{array}{c}\text { Shift } \mu=4000 \mathrm{rad} / \mathrm{sec} \\
q=4 \text { tral vectors }\end{array}$} \\
\hline & Iterations & Time (sec) & Iterations & Time (sec) \\
\hline 1 & 10 & 20 & 7 & 7 \\
\hline 2 & 10 & 69 & 7 & 24 \\
\hline 4 & 11 & 342 & 7 & 122 \\
\hline 8 & 12 & 2255 & 8 & 918 \\
\hline 16 & 12 & 22,105 & 8 & 9499 \\
\hline
\end{tabular}

few frequencies and modes shape for large systems of matrix equations derived from finite element analysis of fluid-structure interactions. The two main advantages for the determinant search method, when compared with a previous approach [1] are:

(1) The current inverse iteration is derived directly from the linear eigenproblem. It is therefore possible to guarantee convergence theoretically.

(2) The current procedure for orthogonalization is exact, whereas the previous procedure was approximate. This is possible because we now iterate on $\mathbf{Z}$ as well as $\mathbf{X}$.

The subspace iteration procedure is new, and is faster than the determinant search algorithm when the matrices become quite large.

In addition, we have derived properties of the eigenproblem from the gyroscopic form of the governing matrix equations. This provides some insight into the physics of the fluid-structure problem. Also, it helped to identify a fundamental difficulty with zero eigenvalues which had not been noted previously.

We feel that several questions are still open. Special steps will have to be taken to remove the rigid body modes from the matrix equations. An efficient procedure for this would be quite useful. Also, because we have transformed our quadratic eigenproblem to a problem which is linear in $\omega$, the convergence of inverse iteration is also linear in $\omega$. If the problem could be recast so that it is linear in $\omega^{2}$, iterations would be significantly faster. Finally, since the subspace iteration is significantly accelerated by shifting, a spectrum slicing search followed by subspace iteration may be worth investigating.
Acknowledgement - This work was supported in part by a Rackham Grant from the University of Michigan.

\section{REFERENCES}

1. L. G. Olson and K. J. Bathe. Analysis of fluid-structure interactions. A direct symmetric coupled formulation based on the fluid velocity potential. Comput. Struct. 21, 21-32 (1985).

2. L. G. Olson and K. J. Bathe, An infinite element for analysis of transient fluid-structure interactions, Engng Comput. 2. 319-330 (1985)

3. L. G. Olson, Finite element model of ultrasonic clearling. $J$. Sound Vibr. (to appear).

4. L. Meirovitch, A new method of solution of the eigenvalue problem for gyroscopic systems. $A I A A \mathrm{Jnl} 12$, 1337-1342 (1974)

5. L. Meirovitch, On the reduction of the eigenvalue problem for spinning axusymmetric structures. $A I A A J n l$ 14, 150-157 (1976).

6. K. K. Gupta. On a combined Stürm sequence and inverse iteration technique for eigenproblem solution of spinning structures. Int. J. Numer. Meth. Engng 7, $509-518$ (1973).

7. W. H. Wittrick and F. W Willams, On the free vibration analysis of spinning structures by using discrete or distributed mass moments. $J$. Sound Vibr. 82 . 1-15 (1982).

8. K. Huseyin, Standard forms of the eigenvalue problems associated with gyroscopic systems $J$. Sound $V i b r 45$, 29-37 (1976).

9. R. H. Plaut, Alternative formulations for discrete gyroscopic eigenvalue problems $A I A A J n l 14,431-435$ (1976).

10. F. Janssens, Solution of linear gyroscopic systems. J. Guidance, Control, Dynam. 5, 92-94 (1982).

11. M. Borri and P. Mantegazza, Efficient solution of quadratic eigenproblems arısing in dynamic analysis of structures. Comput. Meth. appl. Mech. Engng 12, 19-31 (1977).

12 O. A. Bauchau, A solution of the eigenproblem for undamped gyroscopic systems with the Lanczos al-

Table 7. Iterations required to find 10 roots for plate in water problem

\begin{tabular}{|c|c|c|c|c|}
\hline \multirow[b]{2}{*}{$\begin{array}{l}\text { Elements } \\
\text { in plate }\end{array}$} & \multicolumn{2}{|c|}{ Determinant search } & \multicolumn{2}{|c|}{ Subspace iteration } \\
\hline & $\begin{array}{l}\text { Total secant } \\
\text { 1terations }\end{array}$ & $\begin{array}{l}\text { Total inverse } \\
\text { iterations }\end{array}$ & $\begin{array}{c}\mu=0 \mathrm{rad} / \mathrm{sec}, q=36 \\
\text { iterations }\end{array}$ & $\begin{array}{c}\mu=15,000 \mathrm{rad} / \mathrm{sec}, q=18 \\
\text { iterations }\end{array}$ \\
\hline 1 & 83 & 36 & 15 & 24 \\
\hline 2 & 93 & 43 & 21 & 17 \\
\hline 4 & 111 & 45 & 28 & 19 \\
\hline 8 & 121 & 45 & 30 & 14 \\
\hline 16 & 133 & 42 & 34 & 14 \\
\hline
\end{tabular}


gorithm. Int. J. Numer. Meth. Engng 23, 1705-1713 (1980).

13. G. Dietrich, On the efficient and accurate solution of the skew-symmetric eigenvalue problem. Comput. Meth. appl. Mech. Engng 14, 209-235 (1978).

14. A. J. Fricker, A method for solving high-order real symmetric eigenvalue problems. Int. J. Numer. Meth. Engng 19, 1131-1138 (1983).

15. L. Meirovitch, Computational Methods in Structural Dynamics. Sijthoff \& Noordhoff, Rockville, MD (1980).

16. K. J. Bathe, Finite Element Procedures in Engineering Analysis. Prentice-Hall, Englewood Cliffs, NJ (1982)

17. T. J. R. Hughes, The Finite Element Method. Prentice Hall, Englewood Cliffs, NJ (1987).

18. B. N. Parlett, The Symmetric Eigenvalue Problem. Prentice-Hall, Englewood Cliffs, NJ (1980).

\section{APPENDIX. SPECTRUM SLICING}

The spectrum slicing procedure [17], also called the Stürm sequence check [16], follows from Sylvester's inertia theorem [18] for the linear eigenproblem.

$$
\mathbf{E V}=\lambda \mathbf{F V} .
$$

$\mathbf{E}$ and $\mathbf{F}$ are real symmetric matrices, and $\mathbf{F}$ is positive definite. ( $\lambda, V$ is the eigenpair.) The theorem leads to the conclusion that if we write $\mathbf{E}^{*}=\mathbf{E}-\mu \mathrm{F}=\mathbf{L D L}^{T}$ ( $\mathbf{L}$ is a lower triangular matrix and $\mathbf{D}$ is a diagonal matrix), then the number of negative elements in $\mathbf{D}$ is equal to the number of eigenvalues less than $\mu$.

We write our quadratic eigenproblem as

$$
\left[\begin{array}{cc}
\mathbf{0} & \mathbf{M} \\
\mathbf{M} & \mathbf{C}
\end{array}\right]\left[\begin{array}{l}
\mathbf{Z} \\
\mathbf{X}
\end{array}\right]=\frac{1}{\omega}\left[\begin{array}{cc}
\mathbf{M} & \mathbf{0} \\
\mathbf{0} & \mathbf{K}
\end{array}\right]\left[\begin{array}{l}
\mathbf{Z} \\
\mathbf{X}
\end{array}\right]
$$

as $\mathbf{K}$ and $\mathbf{M}$ are assumed to be positive definite. Now we apply a shift $1 / \mu$ ( $\mu$ positive)

$$
\begin{array}{rc}
{\left[\begin{array}{cc}
-(1 / \mu) \mathbf{M} & \mathbf{M} \\
\mathbf{M} & \mathbf{C}-(1 / \mu) \mathbf{K}
\end{array}\right]\left[\begin{array}{l}
\mathbf{Z} \\
\mathbf{X}
\end{array}\right]} \\
=\left(\frac{1}{\omega}-\frac{1}{\mu}\right)\left[\begin{array}{ll}
\mathbf{M} & \mathbf{0} \\
\mathbf{0} & \mathbf{K}
\end{array}\right]\left[\begin{array}{l}
\mathbf{Z} \\
\mathbf{X}
\end{array}\right] .
\end{array}
$$

If we Gauss reduce (factorize) the left-hand side matrix, then the number of positive elements in $D$ should equal the number of $(1 / \omega)>(1 / \mu)$ :

$$
\begin{aligned}
& {\left[\begin{array}{cc}
-(1 / \mu) \mathbf{M} & \mathbf{M} \\
\mathbf{M} & \mathbf{C}-(1 / \mu) \mathbf{K}
\end{array}\right]} \\
& =\left[\begin{array}{ll}
\mathbf{L}_{a} & \mathbf{0} \\
\mathbf{L}_{c} & \mathbf{L}_{b}
\end{array}\right]\left[\begin{array}{cc}
\mathbf{D}_{a} & \mathbf{0} \\
\mathbf{0} & \mathbf{D}_{b}
\end{array}\right]\left[\begin{array}{cc}
\mathbf{L}_{a}^{T} & \mathbf{L}_{c}^{T} \\
\mathbf{0} & \mathbf{L}_{b}^{T}
\end{array}\right] \\
& =\left[\begin{array}{cc}
\mathbf{L}_{\mathbf{a}} \mathbf{D}_{\mathbf{a}} \mathbf{L}_{\mathbf{a}}^{\mathbf{T}} & \mathbf{L}_{\mathbf{a}} \mathbf{D}_{\mathbf{a}} \mathbf{L}_{\mathrm{c}}^{\mathrm{T}} \\
\mathbf{L}_{\mathbf{c}} \mathbf{D}_{\mathbf{a}} \mathbf{L}_{\mathbf{a}}^{\mathbf{T}} & \mathbf{L}_{\mathbf{c}} \mathbf{D}_{\mathbf{a}} \mathbf{L}_{\mathbf{c}}^{\mathbf{T}}+\mathbf{L}_{\mathbf{b}} \mathbf{D}_{\mathrm{b}} \mathbf{L}_{\mathrm{b}}^{\mathbf{T}}
\end{array}\right] \text {. }
\end{aligned}
$$

As $\mathbf{M}$ is positive definite, $\mathbf{D}_{a}$ must always contain $N$ negative entries. (This is reasonable, as the $N$ negative eigenvalues will always be less than any positive $1 / \mu$.) Also, we find that $\mathbf{L}_{b} \mathbf{D}_{b} \mathbf{L}_{b}^{\mathbf{T}}=\mathbf{C}-(1 / \mu) \mathbf{K}+\mu \mathbf{M}$, so that the number of positive entries in $D_{b}$ equals the number of $(1 / \omega)>(1 / \mu)$. As a result, if we factorize

$$
\mathbf{K}^{*}=-\mu(\mathbf{C}-(1 / \mu) \mathbf{K}+\mu \mathbf{M})=\mathbf{K}-\mu \mathbf{C}-\mu^{2} \mathbf{M}, \text { (A6) }
$$

then the number of negative diagonal elements will equal the number of $(1 / \omega)>(1 / \mu)$, i.e. the number of positive eigenvalues $\omega$ which are less than $\mu$. 